\title{
5A's Behavioral Model Framework on Weight Management
}

\author{
Gabrielle Allsup, FNP-BC ${ }^{1^{*}}$ and Melissa R Penkalski, DNP, APRN, CPNP-PC, AE-C ${ }^{2}$ \\ ${ }^{1}$ DNP Student, Missouri State University, USA \\ ${ }^{2}$ Missouri State University, USA
}

*Corresponding author: Gabrielle Allsup, FNP-BC, DNP Student at Missouri State University Doctoral Program of Nursing, 4572 Arabela Dr Las Cruces, NM 88012, USA, Tel: 575-313-9811

\begin{abstract}
Background: Research shows that the United States adult obesity rate is at the highest national rates ever recorded. These numbers have substantially increased since 2008. Obesity-related health conditions include heart disease, stroke, diabetes and certain types of cancer. This disease is preventable, and can be reversed if providers take action now. Research shows that obesity can be managed with diet, exercise and sometimes medications, but education needs to start at the primary care level.
\end{abstract}

Objective: The objectives for this project were to increase provider knowledge and confidence in the evaluation and treatment of obesity utilizing the 5 As behavioral model framework for obesity, which will help reduce obesity rates in our communities.

Methods: This project was implemented at a Federally Qualified Healthcare Center (FQHC) serving a high-risk population for obesity due to local demographics. This FQHC serves a lower socioeconomic population, in addition to the racial and ethnic populations which are more prone to higher rates of obesity. The project consisted of a protocol put into place for the providers and interdisciplinary staff members. An educational session was held for the provider to educate on standard of care guidelines for obesity management as well as the 5 As behavioral model framework for obesity. Both pre- and post-knowledge tests were given at this session. Pre- and post-intervention confidence surveys were also implemented. The interdisciplinary staff members also received an educational session for correct skill techniques for obtaining certain obesity measurements such as blood pressure, weight, height, BMl and waist circumference. A pre- and post-observational evaluation was also implemented within this educational session. The intervention and practice change protocol included having providers evaluate and address individual patient factors contributing to obesity in a 30 minute visit for patients with body mass index $>30 \mathrm{~kg} / \mathrm{m} 2$. All 50 patient participants signed the informed consent forms.
Results: It was observed that the intervention and protocol implemented did increase provider compliance by $84 \%$ shown from descriptive statistics analysis, while knowledge in the evaluation and management of obesity in the primary care clinic setting increased post-intervention by a mean difference of 8.43 based off a parametric t-test. The provider confidence levels both pre- and post- were broken into three different subscales based on agreeance, comfort and rate, and analyzed by either descriptive frequencies or reliability and item analysis based on the Cronbach's alpha using SPSS software. The post confidence mean differences reported were 8.85 in the comfort subscale and a mean difference of 6 in the rate subscale. Therefore, the post mean differences support increased confidence in weight management, while the agreeance subscale of three questions was individually analyzed due to low reliability.

Conclusion: These results will help reduce obesity rates locally and globally by having patients evaluated and managed on their initial visits instead of waiting for a referral to a specialist for treatment. Together, providers can help change the obesity morbidity statistics by educating patients on long term health by working with their primary care providers for that ongoing needed support.

\section{Keywords}

Obesity treatment in adults, Primary care treatment of obesity, Obesity guidelines, 5As model for obesity, PCP obesity treatment, Primary care 5 As model for obesity, Obesity management

Abbreviations
CDC: Centers for Disease Control and Prevention; PCP:
Primary Care Provider; BMI: Body Mass Index; SPSS: Sta-
tistical Package for the Social Sciences; JASP: Jeffrey's
Amazing Statistics Program; COVID-19
in any medium, provided the original author and source are credited. 


\section{Introduction}

Obesity is a complex health issue that affects many Americans. According to the Centers for Disease Control and Prevention [1], the prevalence of obesity was $39.8 \%$ and affected about 93.3 million US adults in 2015-2016 (para. 1). Obesity is a major contributor to many chronic diseases such as hypertension, dyslipidemia, type 2-diabetes, and coronary heart disease, and presents a major public health challenge [2]. The problem with obesity is the lack of evaluation and management in primary care settings. Primary care providers (PCP) are given evidence-based guidelines to follow and help direct treatment for their patients, however evidence shows obesity management in primary care settings still remain suboptimal [3]. The original obesity management guidelines were established in 1998 by the National Heart, Lung and Blood Institute and recommend evaluation and treatment of this condition. From the release of that publication, there have been multiple guideline updates supported by the US Preventative Services Task Force, American Heart Association, American College of Cardiology and The Obesity Society that recommends primary care providers screen patients for overweight and obesity and provide intensive behavioral counseling [4]. Even with these established guidelines in place, only $30 \%$ of patients in primary care are being screened based off of $\mathrm{BMI}$ and treated for this condition. With obesity being a public health concern, the Center for Medicare and Medicaid Services initiated reimbursement in 2011, for PCPs that deliver intensive behavioral therapy to obese patients [4]. It was not until 2013 that the American Medical Association declared obesity as a disease state [5].

Many providers treat chronic health conditions routinely in practice, yet mistreat obesity, even though it is classified as a disease state. Several studies have examined reasons why obesity is underdiagnosed and mistreated and found that providers lack confidence in obesity treatment, lack expertise or training, and had biases towards obesity, in addition were a lack of reimbursement and time constraints in a busy practice [5-7]. Many of these barriers could be addressed with providing education pertaining to the obesity epidemic, the need for change, and reviewing current management guidelines. In addition to the suboptimal treatment provided for obesity management, according to [5] patients stated that they lack awareness of obesity support and assistance from their providers with weight loss.

The $5 \mathrm{As}$ behavioral model for obesity management is a tool that could address these gaps in primary care. The Canadian Obesity Network developed a standardized framework for the use of the 5As in weight counseling which was combined with the Canadian Obesity Guidelines for obesity management.
This framework was adopted from the original smoking cessation counseling tool called the 5As, which include the components ask, assess, advise, agree and assist [8]. Canada's 5As of obesity management program was designed as a step-by-step framework for busy non-specialists, such as primary care providers who manage obesity in their patients [8]. This framework underwent a pilot study and was involved in a larger scale cluster randomized control trial, resulting with recommendations to support the use of the 5As framework facilitates weight management in primary care [8]. This framework provides a positive approach in helping to promote obesity management in primary care settings. This developed framework and associated resources added great benefit to this implementation project. In order to address the many challenges the research has presented about suboptimal obesity management in primary care, increased education to providers is needed, along with a protocol for weight management that is reasonable, quick, and effective for the providers to implement into daily practice. To impact this need a quality improvement project was developed and implemented in a rural primary care setting.

\section{Objectives}

The goal of the project was to implement a protocol that could be utilized for busy providers in the primary care setting to increase their compliance with the standard of care guidelines on obesity management, thus reducing the obesity rates overall. Measureable objectives of this project included the following: At least $85 \%$ provider and $90 \%$ interdisciplinary attendance rates to the educational sessions: significant increases on the post provider knowledge and confidence survey scores: Increased post-observational evaluation scores on the interdisciplinary educational skill session: At least $75 \%$ of patients report the supportive tools and resources having been a positive influence on current goals and habits: At least $90 \%$ of patients had a follow up appointment scheduled prior to leaving the building, and at least $50 \%$ of the patients had a documented plan of care for weight loss/management and/or documentation of the use of the $5 \mathrm{As}$ model used in the chart.

\section{Methods}

To meet ethical considerations the project was approved by the university's institutional review board. June 25, 2020. Over a four month implementation period, 50 participants meeting inclusion criteria participated in the QI project. Inclusion criteria included adult patients over 18 years of age and having had a body mass index of at least $30 \mathrm{~kg} / \mathrm{m}^{2}$, which is classified as obesity. These patients were recruited by the medical assistants asking if they were currently trying to lose weight, interested in weight management, or wanted to participate in a QI project. Additional recruitment 
methods included posters that were posted in the lobby, directing interested patients to mention interest when brought back for vital sign assessment. However, if patients initiated interest, they still needed to meet a $\mathrm{BMI}>30 \mathrm{~kg} / \mathrm{m}^{2}$ to qualify. To assist with recruitment, targeted screening was done prior to the appointment in order to target possible candidates for participation. Patients who agreed to participate and who met inclusion criteria then were scheduled for a 30-minute weight management visit with their provider. At the start of this visit, patients were given written informed consent forms to sign. These visits had a standard protocol of resources and information to be discussed with the patient following the 5As weight management model. Additional resources were also available to use with the patient. After the patient-provider visit, a confidential post visit survey was given to the patients to fill out in order to help determine compliance, satisfaction, usefulness of resources, and likeliness to change current habits after the educational visit. Following the post-survey, the patients were scheduled for a four week follow up appointment. The scheduling of the follow up appointment served as the last step to complete the 5As framework as well as serve as a measureable objective for the project. The provider was responsible for documenting in the patient chart the diagnosis of obesity, the plan of care, and use of the 5As behavioral model framework and follow up care plan. This documentation in the chart also served as measures to ensure provider compliance with the protocol.

In order to get the providers and interdisciplinary staff members educated on the protocol, a few steps took place. Seven medical providers including six nurse practitioners and one physician assistant underwent an educational session which included education on obesity, reviewed current standard of care guidelines for primary care management on obesity, as well as education on the 5 As framework on obesity management. In this 1.5 hour long session, a pre- and post-knowledge test was given over the 5As framework, as well as a pre-confidence likert survey, to determine baseline provider confidence levels in treating obesity. This educational session was done over a lunch period during a scheduled work day. Attendance was taken during this session in order to help meet the project objectives.

The interdisciplinary staff members included five medical assistants and four nurses who went through a similar process; however their 45 minute educational session was geared towards proper skills and techniques for obtaining patient measurements. These included measuring blood pressure both manually and with an automatic machine, height, weight, waist circumference and correct and timely documentation in the chart. These are very important measurements to obtain in order for the providers to do their job in assessing, evaluating and treating obesity. During this session, both a pre and post observational rating likert survey based was completed by the researcher. Again, attendance was taken in order to help meet project objectives.

Status reports were provided weekly and the researcher was available to address any concerns or questions from the staff.

Bi-weekly emails were sent out to both medical assistants and providers as well for updates on the project status.

\section{Results}

The data was analyzed using SPSS and JASP software. The researcher teamed up with the statistical management team from university in order to analyze the data and compile the results. When looking at each objective, it was important to ensure reliability of each survey.

Prior to conducting analyses, the confidence and knowledge data were screened to assess accuracy, missing data, outliers and the violation of assumptions for the confidence subscales agreeance, comfort and rate. The data appeared to be accurate. There were no univariate outliers found when examining the z-scores with a cut-off score of 3 . When assessing univariate normality, the Shapiro-Wilkes values for both confidence comfort summary scores and knowledge showed that normality was met; where-as the assumption for normality was not met for the confidence rate summary scores due to the $p$-values being less than 0.001 . Therefore, both parametric and non-parametric analyses were run to compare the pre- and post-test scores as indicated.

The attendance rates were resulted by means of descriptive frequencies for both providers and interdisciplinary staff members. The objective was met for both providers and interdisciplinary staff as there was a $100 \%$ attendance rate for the educational sessions.

Provider knowledge survey scores were analyzed by running a parametric paired samples t-test to assess differences in the pre and post summary scores. This test showed a significant increase in the mean score from pre to post which suggests that provider knowledge was increased post-educational session $(t(6)=8.26, p<.001$, $d=3.12$ ). Table A1 shows the knowledge pre- $\&$ postmeans and standard error values.

A reliability analysis was computed to determine the psychometric properties of the confidence subscales; agreeance, comfort and rate. The agreeance subscale was found to not be reliable due to Chronbach's alpha being low, and due to limited questions influencing the overall scale no items could be removed. Therefore, the agreeance subscale will have all three questions individually assessed with frequencies. Furthermore, both the comfort and rate subscales were deemed 
reliable, but could be improved with removing certain items (questions $7 \& 13$ in the comfort subscale and question 8 in the rate subscale). These items were removed and the scales were recalculated and found reliable. See Table A2 for Chronbachs' alpha and ranges if items were dropped for each subscale.

The provider agreeance subscale of the confidence survey of three questions was assessed by individual descriptive frequencies showing the percentages of provider answers, see (Table A3, Table A4, and Table A5). The questions were based on a Likert scale of disagree to strongly agree. Overall, this subscale showed that post-intervention providers changed their answers to " $100 \%$ strongly agree that obesity management is an important part of primary care". "Additionally, 14.28\% changing their answers to either agree or strongly agree with the statement about being motivated to learn more about obesity prevention and management."

A paired samples t-test was run to assess the differences in the pre and post confidence subscales of both rate and comfort. The comfort pretest scores were significantly lower than that of the posttest scores $(t(6)=$ 4.84, p. $=0.003, d=1.83$ ). Therefore, provider participants had an increase in confidence in the comfort subscale after the intervention. A Wilcoxon Signed-rank analysis was run to help determine the difference between the rate subscale pre and posttest scores. This analysis showed that the pretest scores were also significantly lower than that of the rate posttest scores $(t(6)=5.86, p=$ $0.001, d=2.22 ; z(6)=0.00, p=0.020, r=1.00)$. Therefore, provider participants had an increase in confidence in the rate subscale after the intervention. See Table A6 for both the average scores and standard error.

The interdisciplinary staff member's data was also screened to assess accuracy, missing data, outliers and violation of assumptions for the pre- and postobservational evaluation of skill and techniques for measuring the following subscales; manual blood pressure, automatic blood pressure, weight, height, waist circumference and documentation of measurements in the chart. Both parametric and nonparametric tests were used to analyze both pre- and post- data collection. A paired samples t-test was run to assess differences in pre- and post- summary scores of the interdisciplinary staff's skills and techniques. All skills assessed including manual BP, automatic BP, height and weight showed a significant increase in observed technique ranking. The following results show how significant posttest increases are for each subscale; Manual BP (t (8) $=11.72, p<0.001, d=3.91$, Automatic $\mathrm{BP}(\mathrm{t}(8)=13.60, \mathrm{p}<0.001, \mathrm{~d}=4.52)$, height $(\mathrm{t}(8)=9.93$, $p<0.001, d=3.28)$ and waist circumference $(t(8)=9.60$, $p<0.001, d=3.20$. The weight subscale was analyzed with both a paired t-test and a Wilcoxon Signed-rank analysis due to the assumption of normality not being met due to the $p$-value being less than 0.001 , like the other subscales. This analysis concluded that the weight pretest summary scores were significantly lower than that of the posttest scores $(t(8)=15.78, p<0.001, d$ $=5.26 ; z(8)=0.00, p=0.006, r=1.00)$. Therefore, the interdisciplinary staff members had an improvement in skill and technique after the educational intervention. In the documentation subscale, all staff members scored the highest available points, indicating improvement and compliance with documentation skills. Interdisciplinary staff members' means summary scores and standard error values for each subscale can be found in Table A7.

In order to determine the results from the patientprovider post-visit satisfaction survey, descriptive frequencies were utilized. Satisfaction with the visit and the resources utilized at the patient-provider visit was rated on a Likert scale from dissatisfied to satisfy. The results showed that $92 \%$ of the participants were satisfied with the visit. In addition, $80 \%$ found the education and resources provided were useful. Another important finding, even though it was not a planned objective to measure, was analyzing if the visit influenced the likelihood of the patient participants to change their current habits in diet, physical exercise and following up with the provider. We were able to capture this information from the data and it is presented in Table A8 for each percentage response to the likelihood of changing current habits.

Post-implementation chart audits were conducted in order to analyze compliance of the protocol from the providers. Descriptive frequencies were used to assess the following; patients had a follow up appointment scheduled; current diagnosis of obesity in the chart, and a documented plan of care for weight management and follow up. Table A9 shows the results from the main chart audit questions to help determine provider compliance. The results showed: $92 \%$ of patients had a follow up appointment scheduled, while $80 \%$ of patients had a diagnosis of obesity in their chart, $84 \%$ of patients had a plan of care and follow up documented. Therefore, these results prove that the objectives were met and at least $90 \%$ of patients had a follow up appointment scheduled, and at least $50 \%$ of the patients had a documented plan of care for weight loss/ management and/or documentation of the use of the 5 As model used in the chart resembling compliance on the provider side.

\section{Discussion}

Key finding from this QI project revealed that it was cost effective and beneficial in a primary care setting. Despite research recommending evaluation and treatment of obesity per evidence-based guidelines, rates of screening and counseling in the primary care setting were found to be only $30 \%$ [4]. In addition, [9] found only $25 \%$ of physicians and nurse practitioners calculated BMIs in the last 30 days during assessments in patients with weight management issues. Much improvement is needed and the results of this QI project 
show how improvements can be made in the primary care setting to follow the obesity management guidelines and increase compliance. In addition, this QI project was able to improve provider knowledge, enhance patient satisfaction, and improve patient willingness to change their current habits due to the patient-provider visits that took place. These are all significant findings that can assist providers help their patients take control of their weight issues, which also affect other aspects of their lives. This implementation project protocol could ensure patients are receiving adequate weight management counseling, thus reducing obesity rates in the United States. This QI project is cost effective, shown to be beneficial, and uses the 5As behavioral model framework. This framework has shown previous success rates and suggests that the framework facilitates weight management in the primary care setting [8]. Therefore, utilization of this established framework enabled the providers' minimal change in practice and allowed for easy implementation with resources and supportive tools. Therefore, implementing this protocol into any primary care setting can be beneficial without excessive change in the current routine or excessive cost.

\section{Limitations}

Limitation of this quality improvement project were that it was implemented during the COVID-19 pandemic, therefore resulting in low sample size and caused the need to implement changes throughout the project to ensure adequate patient participation. COVID-19 affected the patient population seen in the clinic due to most of the visits in person being "sick visits" as compared to routine follow ups. This change impacted the project because sick visits did not have their weight and height measured as the clinic was trying to reduce patient exposure of the virus. This therefore, automatically limited those patients from participation due to the BMI factor not being satisfied and the project requirement for recruitment was based off a BMI of > $30 \mathrm{~kg} / \mathrm{m}^{2}$. Slow recruitment of patients was addressed by the researcher pre-screening the providers' weekly schedule for possible recruitments based on BMI history. This helped the medical assistants focus on specific patients to recruit. In addition, additional limitations were a short implementation period of four months and a small sample size. Researcher bias is also a limitation as the researcher was intricately involved in the recruitment and implementation process with the patients, therefore possibly influencing the positive outcome of the results.

\section{Conclusion}

Obesity is a public health concern with the prevalence of obesity at $42.4 \%$ in 2017-2018 [10]. These alarming incidences and being declared a disease state from the American Medical Association in 2013, support the need changes to be made. The stigma and bias' of obesity discussions need to be limited, and modified in order for the patients to receive evidencebased quality of care. Not only does obesity affect that individual person, obesity affects our nation's health and annual medical costs of $\$ 147$ billion dollars in 2008 [10]. Obesity is a marker for other co-morbidities, but yet it is preventable. With primary care providers taking charge and changing the norm in their clinics by adhering to a protocol and having weight management discussions, providers can help their patient's achieve their best health and optimize their quality of life. Implementing a protocol including the 5As behavioral model framework, can increase provider knowledge and confidence levels in evaluation and management of obesity, thus increasing compliance with the standard guidelines, therefore improving quality of care for our patients, which ultimately can help reduce the rates of obesity in America.

\section{Acknowledgements}

I would like to thank my primary care clinic Ben Archer Health Center for allowing the conduction of this quality improvement project as well as all the staff members who participated. A special thanks to the patient participants that made this project possible, even during the COVID-19 pandemic. Thank you to my clinical preceptor at Ben Archer Health Center, as well as my Clinical Chair at Missouri State University for helping to guide me through this process.

\section{References}

1. Centers for Disease Control and Prevention [CDC] (2018) Adult Obesity Facts.

2. Jensen MD, Ryan DH, Apovian CM, Ard JD, Comuzzie AD, et al. (2014) 2013 AHA/ACC/TOS guideline for the management of overweight and obesity in adults. Journal of the American College of Cardiology 63: 2985-3023.

3. Turner M, Jannah N, Kahan S, Gallaher C, Dietz W (2018) Current knowledge of obesity treatment guidelines by health care professionals. Obesity 26: 623-781.

4. Fitzpatrick SL, Wischenka D, Appelhans BM, Pbert L, Wang $M$, et al. (2016) An evidenced-based guide for obesity treatment in primary care. The American Journal of Medicine 126: 115e.2-155e.7.

5. Barnes ER, Theeke LA, Mallow J (2015) Impact of the provider and healthcare team adherence to treatment guidelines (PHAT-G) intervention on adherence to national obesity clinical practice guidelines in primary care centre. $\mathrm{J}$ Eval Clin Pract 21: 300-306.

6. Hayes S, Wolf C, Labbe S, Peterson E, Murray S (2017) Primary health care providers' roles and responsibilities: A qualitative exploration of 'who does what' in the treatment and management of persons affected by obesity. Journal of Communication in Healthcare 10: 47-54.

7. Turner LR, Harris MF, Mazza D (2015) Obesity management in general practice: does current practice match guideline recommendations? Med J Aust 202: 370-372.

8. Obesity Canada (2019) 5As of obesity management.

9. Vasudevan DA, Northrup TF, Mandayam S, Bamidele OO, Stotts AL (2017) Impact of physician training on diagnosis and counseling of overweight and obese Asian patients. J Racial Ethn Health Disparities 4: 322-328.

10. Centers for Disease Control and Prevention [CDC] (2021) Adult obesity facts. 


\section{Appendix A}

Table A1: Means \& SE for provider pre and post summary scores.

\begin{tabular}{|l|l|l|l|l|}
\hline & Pre & & Post & \\
\hline \multirow{2}{*}{ Provider Knowledge } & X & SE & X & SE \\
\hline & 8.714 & 2.29 & 17.14 & 2.34 \\
\hline
\end{tabular}

Table A2: Confidence Subscale Chronbach's Alpha and range if item is deleted.

\begin{tabular}{|l|c|l|}
\hline & Chronbach's Alpha & Range of Alpha if items are deleted \\
\hline Agreeance & -0.125 & $-0.125-0.824$ \\
\hline Comfort & 0.839 & $0.801-0.863$ \\
\hline Updated Comfort (Excluding questions 7 \& 13) & 0.874 & $0.847-0.874$ \\
\hline Rate & 0.774 & $0.693-0.836$ \\
\hline Updated Rate (Excluding question 8) & 0.836 & $0.761-0.836$ \\
\hline
\end{tabular}

Table A3: Percent responses from provider agreeance subscale confidence survey- Question 1.

\begin{tabular}{|l|l|l|}
\hline & Pre $\%$ & Post $\%$ \\
\hline Agree & $85.71 \%$ & 0 \\
\hline Strongly & $14.28 \%$ & $100 \%$ \\
\hline
\end{tabular}

Table A4: Percent responses from provider agreeance subscale confidence survey- Question 2.

\begin{tabular}{|l|l|l|}
\hline & Pre $\%$ & Post \% \\
\hline Disagree & $85.71 \%$ & $85.71 \%$ \\
\hline Neutral & $14.28 \%$ & $14.28 \%$ \\
\hline
\end{tabular}

Table A5: Percent responses for provider agreeance subscale confidence survey-Question 3.

\begin{tabular}{|l|l|l|}
\hline & Pre $\%$ & Post \% \\
\hline Neutral & $14.28 \%$ & 0 \\
\hline Agree & $71.42 \%$ & $85.71 \%$ \\
\hline Strongly Agree & $14.28 \%$ & $14.28 \%$ \\
\hline
\end{tabular}

Table A6: Means and SE for Confidence subscales Comfort and Rate.

\begin{tabular}{|l|l|l|l|l|}
\hline & Pre & & Post & \\
\hline \multirow{2}{*}{ Comfort } & $\mathrm{X}$ & SE & $\mathrm{X}$ & SE \\
\cline { 2 - 5 } & 32 & 1.95 & 40.85 & 0.59 \\
\hline Rate & 16.57 & 1.36 & 22.57 & 0.57 \\
\hline
\end{tabular}

Table A7: Means \& SE for subscale summary scores for interdisciplinary staff members evaluation survey.

\begin{tabular}{|c|llll|}
\hline & Pre & \multicolumn{3}{c|}{ Post } \\
\hline & X & SE & X & SE \\
\cline { 2 - 5 } Manual BP & 52.11 & 0.564 & 59.00 & 0.645 \\
\hline Automatic BP & 37.77 & 0.465 & 41.55 & 0.377 \\
\hline Weight & 22.66 & 0.167 & 26.78 & 0.324 \\
\hline Height & 28.55 & 0.242 & 32.89 & 0.484 \\
\hline Waist & 21.89 & 0.389 & 26.44 & 0.503 \\
\hline Documentation & 10.22 & 0.465 & 15.00 & 0.000 \\
\hline
\end{tabular}

Table A8: Patient assessment survey questions on post patient-provider visit (Todays visit).

\begin{tabular}{|l|l|l|l|}
\hline & Unlikely & Undecided & Likely \\
\hline Likelihood to change diet after today & $0 \%$ & $32 \%$ & $68 \%$ \\
\hline Likelihood to change physical activity after today & $0 \%$ & $32 \%$ & $68 \%$ \\
\hline Likelihood to return for follow up appointment & $0 \%$ & $42 \%$ & $58 \%$ \\
\hline
\end{tabular}

Table A9: Provider compliance in chart audit documentation.

\begin{tabular}{|ll|}
\hline & $\%$ of compliance \\
\hline Patient follow up scheduled & $92 \%$ \\
Diagnosis of obesity in chart & $80 \%$ \\
\hline $\begin{array}{l}\text { Documentation of plan of care and } \\
\text { follow up }\end{array}$ & $84 \%$ \\
\hline
\end{tabular}

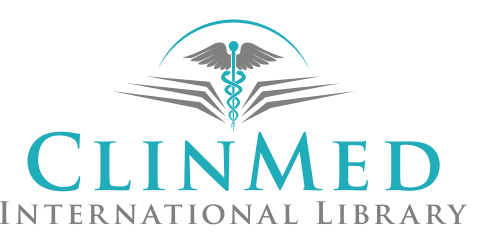

